\title{
Thermodynamic Properties of Spherically-Symmetric, Uniformly-Accelerated Reference Frames
}

\author{
Chao-Guang Huang 11 and Jia-Rui Sun', \\ Institute of High Energy Physics, Chinese Academy of Sciences, \\ P.O. Box 918-4, Beijing 100049, China
}

\begin{abstract}
We aim to study the thermodynamic properties of the spherically symmetric reference frames with uniform acceleration, including the spherically symmetric generalization of Rindler reference frame and the new kind of uniformly accelerated reference frame. We find that, unlike the general studies about the horizon thermodynamics, one cannot obtain the laws of thermodynamics for their horizons in the usual approaches, despite that one can formally define an area entropy (Bekenstein-Hawking entropy). In fact, the common horizon for a set of uniformly accelerated observers is not always exist, even though the Hawking-Unruh temperature is still well-defined. This result indicates that the Hawking-Unruh temperature is only a kinematic effect, to gain the laws of thermodynamics for the horizon, one needs the help of dynamics. Our result is in accordance with those from the various studies about the acoustic black holes.

PACS number 04.70.Dy

Key words: uniformly accelerated reference frame, Hawking-Unruh temperature, entropy of horizon
\end{abstract}

\section{Introduction}

People used to studying physics in the inertial reference frame. However, when the accelerated reference frame is involved, the physics becomes rather different. A well-known example is the vacuum state in a flat spacetime. The vacuum state of a quantum field in a flat spacetime is independent of the choice of inertial frames due to the Pioncaré symmetry. Nevertheless, an accelerated detector will register particles in the vacuum of Minkowski spacetime, i.e., detect a background temperature of the flat spacetime [1, which indicates that the concept of quantum state is dependent of the choice of reference frames. Since the Rindler reference frame 2 has a temperature, it is naturally to study its thermodynamic properties. Some works have been done on this topic [3, 4, and their results incline to the existence of horizon entropy and the laws of thermodynamics for the Rindler horizon. The aim of the present paper is to study whether there exists thermodynamics for the horizon of the reference frame with uniform acceleration. Now that the definition of entropy for the horizon needs the horizon area be finite, we focus on discussing the thermodynamic properties of the spherically symmetric generalization of both Rindler reference frame and the new kind of uniformly accelerated reference frame recently proposed in [5. Both cases describe a set of uniformly accelerated observers in which all the observers initially locate at the same sphere with some fixed radius, and move (outward or inward) along the radius with the same 4-velocity and 4-acceleration. When the set of observers moving outward, each of them has his own event horizon. Each 2-dimensional horizon is a plane which divides the space into two parts: one has causal connection to the observer, another does not have, corresponding to the causal or noncausal part, respectively. The intersection of all the noncausal parts is a 3-dimensional ball in space. Its boundary, a sphere, just acts as the 2-dimensional common event horizon for these observers, i.e., all of them locate outside the sphere cannot 'see' the inside of the sphere. Viewed in this way, we define it as the 2-dimensional event horizon for the set of uniformly accelerated observers. In addition, one may formally define an area entropy for it. When the set of observers moving inward, each of them also has his own event horizon, and each 2-dimensional horizon is again a plane dividing the space into two parts. But the

\footnotetext{
${ }^{1}$ huangcg@ihep.ac.cn

${ }^{2}$ sun@ihep.ac.cn
} 
intersection of those noncausal parts is now empty! So there is no common event horizon for this set of uniformly accelerated observers. In other words, the event horizon for this set of observers does not exist. If one persisted in introducing an area entropy, one immediately find that the area entropy becomes ill-defined. Meanwhile, one should note that the Hawking-Unruh temperature is always well-defined in all the situations. That is to say, one cannot assign an area entropy to the event horizon for the set of uniformly accelerated observers because the horizon is not always exist in these reference frames. The studies about the spherically symmetric generalization of Rindler reference frame and the new kind of uniformly accelerated reference frame both indicate that the Hawking-Unruh temperature is only a kinematic effect, to gain the laws of thermodynamics for the event horizon, one needs the help of dynamics. This point is in accord with the previous studies about the acoustic black holes [6, 7, 8, and gives examples that are different to the general horizon thermodynamics (e.g., Rindler spacetime, de Sitter spacetime and Friedmann-Robertson-Walker (FRW) universe) which have been widely studied by many authors [3, 4, 9, 10, 11, 12, 13, 14.

The arrangement of this paper is in the following way: we begin in the next section to give a brief review to the Rindler reference frame, Møller reference frame and a new kind of reference frame with uniform acceleration. In section 3, we pay our special attention to their spherically symmetric generalizations and study their thermodynamic properties. Finally, we will make the conclusions and discussion.

Throughout the paper, we consider the 4-dimensional spacetime and use the natural units $\hbar=$ $c=G=k_{B}=1$.

\section{Unifomly accelerated reference frames}

\subsection{Rindler and Møller reference frame}

The Rindler reference frame is often expressed in Cartesian coordinates [2, 15],

$$
d s^{2}=-x^{2} d t^{2}+d x^{2}+d y^{2}+d z^{2} .
$$

For later convenience, we make a little change of the above metric

$$
d s^{2}=-g^{2} x^{2} d t^{2}+d x^{2}+d y^{2}+d z^{2},
$$

where $g$ is a constant. A static observer with a 4 -velocity $U^{\mu}=\{1 /(g x), 0,0,0\}$ possesses a 4acceleration $a^{\mu}=U^{\nu} \nabla_{\nu} U^{\mu}=\{0,1 / x, 0,0\}$. Its magnitude is $a \equiv\left(a^{\mu} a_{\mu}\right)^{1 / 2}=1 / x$. It is clear to see that at each fixed space point $\{x, y, z\}$, the acceleration is a constant, but the acceleration varies point by point, namely, different observer possesses different acceleration.

By making the coordinates transformations of the Rindler reference frame

$$
t \rightarrow \tilde{t} \text { and } x \rightarrow(1+g \tilde{x}) / g .
$$

Eq.(2) changes to the Møller reference frame 16]

$$
d s^{2}=-(1+g \tilde{x})^{2} d \tilde{t}^{2}+d \tilde{x}^{2}+d y^{2}+d z^{2} .
$$

Similar calculation shows that the magnitude of the 4-acceleration for a static observer with $U^{\mu}=$ $\{1 /(1+g \tilde{x}), 0,0,0\}$ at fixed $\tilde{x}, y, z$ is

$$
a=\frac{g}{1+g \tilde{x}}
$$

which is also a constant. However, different static observer possesses different acceleration in Møller reference frame, too.

The Rindler horizon has a temperature $T_{H}$ relating to its 'surface gravity'

$$
\kappa=\lim _{x \rightarrow x_{H}} V a=g
$$


by the known relation $T_{H}=\kappa / 2 \pi$ between surface gravity and Hawking-Unruh temperature,

$$
T_{H}=\frac{g}{2 \pi},
$$

where $V=\sqrt{-g_{00}}$ is the redshift factor. The Hawking-Unruh temperature corresponding to the Møller reference frame is the same as Rindler's.

\subsection{New uniformly accelerated reference frame}

The new kind of uniformly accelerated reference frame was first proposed in $[5]$ in Cartesian coordinates

$$
d s^{2}=-d T^{2}+2 \sinh (a T) d T d X+d X^{2}+d y^{2}+d z^{2},
$$

where $a$ is a constant, for a 'static' observer with 4 -velocity $U^{\mu}=\{1,0,0,0\}$, it is easy to see that his 4-acceleration is $a^{\mu}=\{a \tanh (a T), a \operatorname{sech}(a T), 0,0\}$, and the magnitude of $a^{\mu}$ is just $\left(a^{\mu} a_{\mu}\right)^{1 / 2}=a$ which is indeed a constant acceleration. In addition, it has been shown in [5] that the equation of motion reduces to the standard second law of mechanics in the Newtonian approximation, whereas, the second law of mechanics in Rindler reference frame does not have this property.

By a Wick rotation of the time coordinate

$$
T \rightarrow \tau=i T
$$

in Eq.(8), we get the imaginary time period $\beta=2 \pi / a$, which leads to the Hawking-Unruh temperature of the horizon

$$
T_{H}=\frac{1}{\beta}=\frac{a}{2 \pi} .
$$

If one uses the relation between temperature and 'surface gravity', he or she will get the same Hawking-Unruh temperature.

\section{Thermodynamic Properties of Spherically Symmetric Ref- erence Frames}

As we have mentioned in Introduction, the planar Rindler reference frame and the new kind of uniformly accelerated reference frame is not very convenient for studying their thermodynamic properties, e.g., defining the entropy for the horizon requires the horizon to have a finite surface. So we will generalize these uniformly accelerated reference frames in section $\mathbf{2}$ into spherically symmetric cases.

\subsection{Spherically symmetric generalization of Rindler reference frame}

Firstly, we study the generalized Rindler reference frame. Recall that the 4-dimensional Minkowski spacetime in spherical coordinates is

$$
d s^{2}=-d t^{2}+d r^{2}+r^{2}\left(d \theta^{2}+\sin ^{2} \theta d \phi^{2}\right) .
$$

After using the following coordinates transformations

$$
\rho-\rho_{0}= \pm\left[\left(r-r_{0}\right)^{2}-t^{2}\right]^{1 / 2} \text { and } \quad \eta=\frac{1}{a} \tanh ^{-1}\left[t /\left(r-r_{0}\right)\right],
$$

the line-element (11) becomes

$$
d s^{2}=-a^{2}\left(\rho-\rho_{0}\right)^{2} d \eta^{2}+d \rho^{2}+r^{2}(\eta, \rho)\left(d \theta^{2}+\sin ^{2} \theta d \phi^{2}\right),
$$


where $r(\eta, \rho)$ means $r$ is the function of $\eta$ and $\rho$. A simple calculation shows that Eq.(13) is just the generalized Rindler reference frame in spherically symmetric coordinates, since the 4 -acceleration is $a^{\mu}=\left\{0,\left(\rho-\rho_{0}\right)^{-1}, 0,0\right\}$ for a static observer with 4-velocity $U^{\mu}=\left\{a^{-1}\left(\rho-\rho_{0}\right)^{-1}, 0,0,0\right\}$, and the corresponding Hawking-Unruh temperature is $T_{H}=a /(2 \pi)$. This reference frame describes a set of observers locate at the same sphere with some fixed radius $r$, and all of them move (outward or inward) along the radius with the same $U^{\mu}$ and $a^{\mu}$. It can also be seen from the calculation that the ' + ' in Eq. (12) corresponds to observers moving outward along the $r$ axis with uniformly acceleration, while '-' corresponds to the inward moving case. Note that each observer has his own event horizon, and like the planar Rindler case, each 2-dimensional horizon is a plane tangent to the sphere $\rho=\rho_{0}$. To find the common event horizon for this set of uniformly accelerated observers, we study the following two situations.

When this set of uniformly accelerated observers moving outward along $r$ axis, one has $r=r_{0}+t$. For each observer, there is a 2-dimensional event horizon which divides the space into a causal part and a noncausal part. The intersection of all these noncausal parts form a 3-dimensional ball, light signals emitted in the ball cannot affect anyone of the observers since these observers locate at some $r>r_{0}$ (i.e., outside the sphere). That is to say, the boundary of the 3-dimensional ball just acts as the common event horizon for these observers, namely, they have the same event horizon. Thus we define the 2-dimensional sphere as the event horizon for this set of uniformly accelerated observers. If the Bekenstein-Hawking entropy

$$
S=\pi\left(r_{0}+t\right)^{2}
$$

is assigned for the horizon, it seems not to violate the second law of thermodynamics as the time $t$ evolves.

When these observers moving inward to the origin $r=0$ along $r$ axis with uniform acceleration $a^{\mu}, r=r_{0}-t$. One should pay attention that in this situation, although for each observer there is an event horizon, for all the observers, there will be no common event horizon at all! This is because the spatial section of the event horizon for each observer is a 2-dimensional plane tangent to the sphere at $\rho=\rho_{0}$ (i.e., $r=r_{0}-t$ ), which separates the space into two parts. In the causal part, a light signal emitted in this region can affect the observer eventually. While in the noncausal part, no signal can affect the observer. The intersection of these noncausal parts is empty. However, the 'envelop' of these 2-dimensional plane at the same sphere is also a sphere, if its radius $r=r_{0}-t$ is positive. Notice that all of the uniformly accelerated observers locate at some $r<r_{0}-t$ since $\rho<\rho_{0}$ in this case, that is to say, these observers are within the sphere, which indicates that light signals emitted from any place can always affect some observers. Namely, the 2-dimensional sphere cannot be regarded as the common event horizon for this set of uniformly accelerated observers. Consequently, there is no such an area entropy for the horizon. If one persisted in assigning an area entropy to the 2 -dimensional sphere by analogy with the black hole thermodynamics, i.e., the entropy $S$ was a quarter of the area $A$ of the sphere, where $A=\left.4 \pi r^{2}\right|_{\rho=\rho_{0}}=4 \pi\left(r_{0}-t\right)^{2}\left(r_{0}\right.$ is a constant), the entropy

$$
S=\pi\left(r_{0}-t\right)^{2}
$$

would decrease for the isolated system as the time $t$ evolves when $r_{0}>0$. Obviously, it will violate the second law of thermodynamics. Thus, the entropy of horizon for inward, uniformly accelerated observers is ill-defined.

\subsection{Spherically symmetric generalization of the new uniformly acceler- ated reference frame}

Now, we turn to the generalized new uniformly accelerated reference frame. By making the following coordinates transformations

$$
t=\frac{1}{a} \sinh (a T) \quad \text { and } \quad r=R+\frac{1}{a}(\cosh (a T)-1)
$$

in Eq.(11), we get a new spherically symmetric coordinates in the Minkowski spacetime

$$
d s^{2}=-d T^{2}+2 \sinh (a T) d T d R+d R^{2}+\left[R+\frac{1}{a}(\cosh (a T)-1)\right]^{2}\left(d \theta^{2}+\sin ^{2} \theta d \phi^{2}\right) .
$$


It can be seen that the first three terms in (8) and (17) are quite similar. This is because the same form of coordinates transformations has been used as in getting (8) from the flat Minkowski spacetime. A short calculation shows that the 4 -acceleration is $a^{\mu}=\{a \tanh (a T), a \operatorname{sech}(a T), 0,0\}$ for a 'static' observer with 4-velocity $U^{\mu}=\{1,0,0,0\}$, and the magnitude of the acceleration $\left(a^{\mu} a_{\mu}\right)^{1 / 2}=a$ is a constant. Therefore, this reference frame just describes a set of uniformly accelerated observers in which all of them initially locate at the same sphere with some fixed radius, and move (outward or inward) along the radius with the same $U^{\mu}$ and $a^{\mu}$.

Since this uniformly accelerated reference frame is described by a metric which is neither static nor stationary, the usual approach to determine the location of its horizon like for the Rindler reference frame and Schwarzschild black hole is not applicable. Instead, we search it following the approach in cosmology. Note that the 3-dimensional event horizon is generated by the null curves. That is, we have

$$
d s^{2}=0
$$

for the generators of the horizon. When the set of observers on a sphere with radius $R$ and moving towards the radial direction, respectively. Eq.(17) becomes

$$
d s^{2}=-d T^{2}+2 \sinh (a T) d T d R+d R^{2}=0,
$$

which can be rewritten as

$$
\frac{d R^{2}}{d T^{2}}+2 \sinh (a T) \frac{d R}{d T}-1=0
$$

this differential equation has two solutions

$$
\frac{d R}{d T}=\exp [-a T] \quad \text { or } \quad \frac{d R}{d T}=-\exp [a T] .
$$

The first solution indicates the future outgoing mode, while the second one indicates the future incoming mode. Consider these observers locate at large enough $R$, one has

$$
R-R_{H}=\int_{R_{H}}^{R} d R=\int_{T}^{\infty} \exp [-a T] d T=\frac{1}{a} \exp [-a T] .
$$

Namely, the 2-dimensional future event horizon is at

$$
R_{H}=R-\frac{1}{a} \exp [-a T]
$$

in the uniformly accelerated reference frame. In particular, at $T=0$,

$$
R_{H}=R-\frac{1}{a}
$$

While $T \rightarrow \infty$, the event horizon will reach its maximum

$$
\lim _{T \rightarrow \infty} R_{H} \rightarrow R_{m}=R
$$

i.e., approach to the position of observer. Obviously, the sets of observers at different radius have different horizons!

The horizon area $A$ is

$$
\begin{aligned}
A & =\int \sqrt{\sigma} d \theta d \phi=4 \pi\left[R_{H}+\frac{1}{a}(\cosh (a T)-1)\right]^{2} \\
& =4 \pi\left[R+\frac{1}{a}(\sinh (a T)-1)\right]^{2}
\end{aligned}
$$

where $\sigma$ is the determinant of the induced metric on the 2-dimensional sphere. 
The Hawking-Unruh temperature of the horizon is easily obtained from the Wick rotation: $T_{H}=$ $a /(2 \pi)$. One may also formally define a Bekenstein-Hawking entropy $S$ for the event horizon by analogy with the black hole entropy, i.e.,

$$
S=\frac{A}{4}=\pi\left[R+\frac{1}{a}(\sinh (a T)-1)\right]^{2} .
$$

Nevertheless, according to the experience we learned from the spherically symmetric generalization of Rindler reference frame, one should take care whether it make sense to assign such an entropy to the event horizon of this new reference frame. Recall that $R$ which comes from the coordinate transformations in Eq.(16) is a constant for some given 'static' observers in the uniformly accelerated reference frame. When $R<a^{-1} \exp [-a T]$ or $R<a^{-1}$, Eq.(23) or Eq.(24) gives the negative value. In both situations, light signals emitted from any place can always affect some observers on the sphere with radius $R$, which means that in these situations, there are no common event horizon for the set of observers. Consequently, no area entropy could be introduced. However, the Hawking-Unruh temperature is always exist for accleratad observers based on Unruh's discussion on the thermal effect of an accelerated detector [1].

The absence of event horizon but meanwhile the existence of Hawking-Unruh temperature is a new property of the spherically symmetric accelerated reference frames including the generalization of Rindler reference frame and the generalization of this new reference frame with 'dynamic' metric. This gives different examples to the general horizon thermodynamics which have been widely studied by many authors $4,9,10,11,13,14$.

\subsection{Hawking-Unruh temperature without area entropy}

What do they tell us? Following the above discussions, both of the two examples: generalized Rindler reference frame and generalized new uniformly accelerated reference frame, seem to give us a hint that the thermodynamic properties of their horizons may be different from those of the general horizon thermodynamics, like the black holes, de Sitter spacetime and FRW universe, in which the Einstein equation is used in getting the laws of thermodynamics. Pay attention that, in obtaining the Hawking-Unruh temperature for the two examples, one indeed does not apply any dynamics at all. Moreover, the Hawking-Unruh temperature is always well-defined even though the common event horizon of the set of uniformly accelerated observers does not exist. So these two examples, both indicate that the appearance of the Hawking-Unruh temperature (or Hawking radiation) is only a kinematic effect but not a dynamic one. This conclusion is in accord with the studies about the acoustic black holes [6, 7, 8, (for a review, see [7). Where in these cases, on one hand, by making an analogy between the sonic field in fluid field and the quantum field in gravitational field, a kind of acoustic metrics which are called the acoustic black holes are obtained, they are of the same form of the Schwarzschild metrics, and then the Hawking radiation is obtained naturally. On the other hand, the fluid flow system has nothing to do with the real black hole system, hence the introduction of entropy to their acoustic horizon is meaningless. In turn, one can learn from these examples that:

a. the Hawking radiation is only a kinematic effect, regardless of the dynamics, e.g., the Einstein equation (which, in fact, can have been seen from Hawking's original derivation [17] [18]); and

$b$. it is only when the dynamics is applied, e.g., the Einstein equation, that one can assign the entropy to the horizon, and hence gain the laws of thermodynamics for the horizon (which can be seen from [19] and has been proved indirectly in [20] to some degree).

In addition, studies about acoustic black holes indicate that the Hawking radiation (or HawkingUnruh temperature) will exist in any Lorentzian geometry with an event horizon [7, 8, but according to the discussion in previous subsections, our examples go further: Hawking-Unruh temperature will exist even when there is no common event horizon for the set of observers with uniform acceleration (though each observer, of course, has his own event horizon).

So far, we have answered the question which we have previously proposed, that is, the existence of Hawking-Unruh temperature is only a kinematic effect, to obtain the laws of thermodynamics for the horizon, one needs the help of dynamics. Since the spherically symmetric generalizations of the 
Rindler reference frame and the new uniformly accelerated reference frame indeed do not involve any dynamics, so assigning the entropy and laws of thermodynamics for their event horizons (if the common event horizon exists) make no sense.

\section{Conclusions and Discussion}

Through the study of thermodynamic properties of the generalizations of Rindler reference frame and a new kind of uniformly accelerated reference frame in spherically symmetric coordinates case, we finally find that there is no laws of thermodynamics for them by applying the usual approaches. Our result, not only supports the studies of acoustic black holes, but also broaden their conclusions to the situation in which the common event horizon is not always exist for the set of uniformly accelerated observers. All of this, seems to indicate one thing that the Hawking-Unruh temperature, which is only a kinematic effect, has no intrinsic connection with the horizon area entropy (BenkensteinHawking entropy), which is a holographic property corresponding to the gravitation. Hence in order to get the thermodynamic laws and further find the microscopic origin of the Benkenstein-Hawking entropy for black holes, the gravitational laws, i.e., the Einstein equation or other quantum theory of gravity should be introduced.

In the end, review that when the initial condition is properly selected to let the horizon make sense, we can see that e.g., the entropy $S$ in Eq.(27) is never decreasing as the time $T$ evolves when $R_{H}$ in Eq.(24) is positive, which implies that something like the generalized second law of thermodynamics is hold for this system [21, 22. Be that as it may, we prefer to take this as merely an analogy but not of the real physical meaning.

Acknowledgments: We are grateful to Prof. H.-Y. Guo for stimulating discussions. JRS would like to thank Prof. R.-G. Cai and X. Li for useful discussions and comments, and thank T. Qiu for making comment on the earlier draft of this paper. This work is supported by NSFC under Grant Nos. 90403023 and 10375087.

\section{References}

[1] W. G. Unruh, "Notes on black-hole evaporation," Phys. Rev. D14, 870 (1976).

[2] W. Rindler, "Kruskal space and the uniformly accelerated frame," Am. J. Phys. 34, 1174 (1966).

[3] T. Padmanabhan, "Classical and quantum thermodynamics of horizons in spherically symmetric spacetimes" gr-qc/0204019. "Thermodynamics of horizons: A comparison of Schwarzschild, Rindler and de Sitter spacetimes," Mod. Phys. Lett. A17, 923 (2002).

[4] H. Culetu, "Is the Rindler horizon energy nonvanishing?" hep-th/0607049.

[5] C.-G. Huang and H.-Y. Guo, "A new kind of uniformly accelerated reference frame", Int. J. Mod. Phys. D15, 1035 (2006), gr-qc/0604008.

[6] W. G. Unruh, "Experimental black-hole evaporation?" Phys. Rev. Lett. 46, 1351 (1981).

[7] M. Visser, "Acoustic black holes: Horizons, ergospheres, and Hawking radiation" Class. Quant. Grav. 15, 1767 (1998), gr-qc/9712010

[8] M. Visser, "Hawking radiation without black hole entropy" Phys. Rev. Lett. 80, 3436 (1998), gr-qc/9712016.

[9] G. W. Gibbons, S. W. Hawking, "Cosmological event horizons, thermodynamics, and particle creation" Phys. Rev. D15, 2738 (1977). 
[10] M. Spradlin, A. Strominger and A. Volovich, "Les Houches lectrues on de Sitter space" hep-th/0110007.

[11] C.-G. Huang, L. Liu and B. Wang, "Thermodynamics of de Sitter universes" Phys. Rev. D65, 083501 (2002).

[12] H.-Y. Guo, C.-G. Huang and B. Zhou, " Temperature at horizon in de Sitter spacetime" Europhys. Lett.72, 1045 (2005), hep-th/0404010

[13] R.-G. Cai, S. P. Kim, "First law of thermodynamics and Friedmann equations of FriedmannRobertson-Walker universe" JHEP 0502, 050 (2005), hep-th/0501055.

[14] T. Padmanabhan, "Cosmological constant: The weight of the vacuum" Phys. Rep. 380, 235 (2003), hep-th/0212290 "Gravity and the thermodynamics of horizons" Phys. Rep. 406, 49 (2005), gr-qc/0311036, and related references therein.

[15] W. Rindler, Essential Relativity, - special, general, and cosmological, 2nd Edition, (SpringerVerlag, New York, 1977)".

[16] C. Møller, The theory of relativity, (Oxford university Press, London, 1952); C. W. Misner, K. S. Thorne and J. A. Wheeler, Gravitation,(Freeman, San Francisco, 1973)."

[17] S. W. Hawking, "Black hole explosions?" Nature 248, 30 (1974).

[18] S. W. Hawking, "Particle creation by black holes" Commun. Math. Phys. 43, 199 (1975).

[19] J. M. Bardeen, B. Carter, and S. W. Hawking, "The four laws of black hole mechanics" Commun. Math. Phys. 31, 161 (1973).

[20] T. Jacobson, "Thermodynamics of space-time: The Einstein equation of state" Phys. Rev. Lett. 75, 1260 (1995), gr-qc/9504004.

[21] J. D. Bekenstein, "Black holes and entropy", Phys. Rev. D7, 2333 (1973).

[22] J. D. Bekenstein, "Generalized second law of thermodynamics in black-hole physics" Phys. Rev. D9, 3292 (1974). 